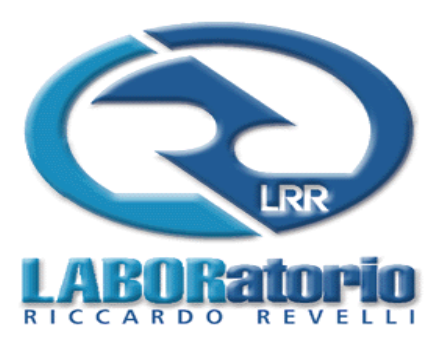

LABORatorio R. Revelli

Centre for Employment Studies

\title{
From Moral to Social Norms and Back
}

\author{
Matteo Richiardi \\ LABORatorio Riccardo Revelli Centre for Employment Studies
}

Version: $02 / 11 / 2005$ 


\begin{abstract}
This paper studies how different behavioural norms affect individual and social welfare in a population with heterogeneous preferences. I assume preferences are private information, and that interactions between individuals do not involve communication, nor bargaining. I first compare two stylized behavioural rules: one states "do to your neighbours what you would like them do to you", also known as Jesus' golden rule, while the other prescribes "don't do to your neighbours what you would not like them do to you", and is attributed to the Jewish rabbi Hillel (I century B.C.). I consider them as an idealization of an imperative and a more liberal approach to social norms. I find that aggregate welfare depends on the distribution of preferences in the society.

A third, more realistic behavioural rule is then introduced, a retaliation strategy that prescribes "do to your neighbours what they have done to you". I show that, if followed by everybody, this strategy leads to the selection of a single behaviour, which becomes established as a social norm. This behaviour leads in general to more inequality, with respect to the Jesus or Hillel rules. However, it is sufficient that a small group (about $1 \%$ ) of the population keeps on playing one of the two moral norms to recover the same social welfare that are obtained when everybody played that moral norm.
\end{abstract}

Keywords $\quad$ : Liberalism, Tit-for-tat, non-market interaction, golden rule JEL Classification : D63, D64, P50

Acknowledgements: A Lagrange fellowship by ISI Foundation is gratefully acknowledged. The author is grateful to Florentin Paladi, who provided a number of computations and helped in getting some of the results of section 3. Matteo Richiardi wishes also to thank Michele Sonnessa, author of JAS - the agentbased simulation platform used in the paper - for his software and programming assistance. 
The Talmud tells that a gentile came to Hillel saying that he would convert to Judaism if Hillel could teach him the whole Torah in the time that he could stand on one foot. Hillel converted the gentile by telling him, "That which is hateful to you, do not do to your neighbor. That is the whole Torah; the rest is commentary. Go and study it."

"And seeing the multitudes, Christ went up into a mountain. And when he was set, his disciples came unto him. And he opened his mouth, and taught them, saying - Do unto others as you would have them do unto you." (Matthew 7:12)

"And if any mischief follow, then thou shalt give life for life, eye for eye, tooth for tooth, hand for hand, foot for foot, burning for burning, wound for wound, stripe for stripe.” (Exodus 21: 23-25)

\section{Introduction}

The emergence of pro-social behaviour in human societies has been the matter of thorough investigations. Two kinds of explanations have been advanced. One builds upon the hypothesis of rational behaviour of self-interested individuals, and stresses the importance of reciprocal altruism (Triver, 1971; Axelrod and Hamilton, 1981): individuals cooperate in exchange of other people's cooperation. The other stresses the importance of cultural (Cavalli-Sforza et al., 1981; Boyd and Richerson, 1985) and genetic (Lumsden and Wilson, 1981; Simon, 1983; Wilson and Dugatkin, 1997; Sober and Wilson, 1998) evolution.

In particular, Fehr and Fischbacher (2004) review evidence that human behaviour is often based on conditional cooperation, i.e. cooperate if other group members cooperate, and defect if other group members defect. They stress the importance of mechanisms such as expectations, reputation and punishment in order to explain the emergence of reciprocal altruism. However, as Gintis (2000) argues, precisely when a group is threatened and is thus most in need of pro-social behaviour the probability of future interactions goes down, together with the incentives for reciprocal altruism.

It is no surprise then that many studies have shown ${ }^{1}$ that people are not only motivated by economic self-interest but also by norms of fairness and reciprocity, that in turn could be explained in terms of evolutionary selection, as sketched above. Religion is one of the mechanisms for strengthening these social norms.

However, although in many cases it is straightforward to identify what is a pro-social behaviour, in general individual preferences are private information. Thus, if player A (the active player) wants to act in an altruistic way towards player $\mathrm{B}$ (the passive player), player A has to guess which action will please the most player B. This point has largely been neglected by the scientific literature, which assumes that the pro-social behaviour is always clearly identified. However, it is present in the religious literature, which generally makes the assumption that, not knowing what your neighbour likes, you should act as if your neighbour were not too different from yourself. This gave rise to a number of "golden rules", of which two prototypes are the Christian and the Jewish golden rule quoted above. The rule stated by Jesus in his Mountain speech (hereafter, J-

\footnotetext{
${ }^{1}$ see the references in the review paper by Fehr and Fischbacher cited above.
} 
rule) prescribes to do what you think is good; the rule stated by Hillel (hereafter, H-rule) prescribes not to do what you think is bad. In the history of philosophy there are many antecedents to both rules. On Jesus side we have the Greek philosophers Sextus, Aristotle, Aristippus and Isocrates, while on Hillel side we have Pittacus, Thales and the Chinese philosopher Confucius.

It is easy to find a flavour of socialism in the J-rule, while the H-rule looks definitely more liberal. ${ }^{2}$ The purpose of this paper is to investigate their implications for aggregate welfare in the simplest possible model. The model is described in section 2, while the results are derived in section 3 . Then, it is then interesting to see what happens if some part of the population departs from the moral norm and plays a sort of tit-for-tat strategy ("what has been done unto you, do it to others"), which as we have seen is after all a very common behaviour. ${ }^{3}$ This extension is dealt with in section 4 and 5. Section 6 summarizes and concludes.

\section{The model}

The model is the same as in the companion paper Richiardi (2005). There are $N$ individuals, who can be in 3 different states (call them Left, Center and Right), and can play 3 actions (again Left, Center and Right). Interaction involves always one active and one passive player ${ }^{4}$. Individuals have preferences over their states: they love one state, they are neutral with respect to another state and they hate the remaining state. When two persons meet, the active player sets the passive player's state according to his action, which in turn is determined by his moral norm.

This identifies only 6 possible combinations. Denote with $p_{1} \ldots p_{6}$ the shares of the population characterized by each combination of preferences, as in table 1 . That is, drawing randomly one individual, she will be of type $i$ with probability $p_{i}$.

\begin{tabular}{cllc}
\hline Type & Loved state & Hated state & Share \\
\hline 1 & Left & Center & $p_{1}$ \\
2 & Left & Right & $p_{2}$ \\
3 & Center & Left & $p_{3}$ \\
4 & Center & Right & $p_{4}$ \\
5 & Right & Left & $p_{5}$ \\
6 & Right & Center & $p_{6}$ \\
\hline
\end{tabular}

Table 1: Distribution of preferences in the population

\footnotetext{
${ }^{2}$ The reader should not consider the results of this paper as a judgement over different religious prescriptions. The two behavioral rules considered are named after Jesus and Hillel for ease of identification, but many other references, aside all the philosophers cited above, could be found.

${ }^{3}$ This rule has also noble origins, reminding the "eye for eye, tooth for tooth" prescription of the Bible and an almost identical prescription to be found in the Hammurabi code ( $8^{\text {th }}$ century B.C.). However, as it will be clear later, the "tit-for-tat" rule used in the paper doesn't allow to address the reaction specifically to the offender, thus the label BT, for "blind tit-for-tat", that will be used.

${ }^{4}$ Agents can play both roles interchangeably.
} 
After each interaction, the passive player gets a payoff of +1 if she is in her loved state, a payoff of 0 if she is in her neutral state, and a payoff of -1 if she is in her hated state. The active player does not get any feedback ${ }^{5}$.

If the active player follows the J-rule, he always plays the action corresponding to his loved state. If he follows the $\mathrm{H}$-rule he randomise between the actions corresponding to his loved and neutral state. An example will clarify.

Suppose two individuals, A and B, meet. Player A is the active one. He hates Left and loves Right (he is thus neutral with respect to Center). Player B is the passive one. She loves Left and hates Right (she is neutral with respect to Center, like player A). Suppose A follows the J-rule. He will play Right, setting B's state to Right. B will then have a payoff of -1 . Suppose on the other hand that A follows the H-rule. He will randomise between Center and Right. The payoff for B could then be either 0 or -1 .

Note that there is no strategic interaction in the model: the passive player's payoff depends on the active player's choice, but the active player's choice does not depend on the passive player in any way. This implies that game-theoretic solution concepts like Nash equilibrium become useless.

Aggregate welfare is defined both in terms of the mean and the coefficient of variation of the payoffs (which denote respectively how rich and how unequal the society is). However, in order to avoid arbitrary choices I do not specify a particular functional form, and report separately the results for the mean and the coefficient of variation. ${ }^{6}$

\section{Results}

It is straightforward to see that when all individuals share the same preferences (polarization) the J-rule is better. In the other extreme case, when preferences are equally distributed in the population (dispersion) and $p_{1}=p_{2}=\ldots=p_{6}=1 / 6$, it is again straightforward to see that the two rules are equivalent, and lead to an average payoff $\pi=0$. Should we infer that the J-rule always dominates the H-rule?

\subsection{Average payoffs}

Consider an active player of type 1 (he loves Left and hates Center), who meets in turn all other (passive) individuals, including himself. If he follows the J-rule, he will play Left, causing a payoff of +1 in $\left(p_{1}+p_{2}\right) N$ individuals, and a payoff of -1 in $\left(p_{3}+p_{5}\right) N$ individuals. Note that there are $\left(p_{1}+p_{2}\right) N$ individuals like him in the population.

Suppose now that everybody meets everybody else both as active and as passive player $^{7}$. The average payoff when everybody plays according to the J-rule is then

$$
\pi_{J}=\left(p_{1}+p_{2}\right)\left(p_{1}+p_{2}-p_{3}-p_{5}\right)+\left(p_{3}+p_{4}\right)\left(-p_{1}+p_{3}+p_{4}-p_{6}\right)+\left(p_{5}+p_{6}\right)\left(-p_{2}-p_{4}+p_{5}+p_{6}\right)
$$

\footnotetext{
${ }^{5}$ We can suppose that he receives a positive payoff deriving from acting accordingly to his moral norm.

${ }^{6}$ In Richiardi (2005) I define aggregate welfare only in terms of the mean, but investigate further extensions of the behavioural rules examined here.

${ }^{7}$ coupling individuals randomly and randomly choosing who is the active and who is the passive player only adds some noise to the results
} 
Similarly, the average payoff with the H-rule is

$$
\pi_{H}=\frac{1}{2}\left(\begin{array}{l}
\left(p_{1}+p_{6}\right)\left(p_{1}+p_{6}-p_{3}-p_{4}\right)+ \\
\left(p_{2}+p_{4}\right)\left(p_{2}+p_{4}-p_{5}-p_{6}\right)+ \\
\left(p_{3}+p_{5}\right)\left(p_{3}+p_{5}-p_{1}-p_{2}\right)
\end{array}\right)
$$

To study the behaviour of $\pi_{J}-\pi_{H}$ I represent the distribution of preferences in the society as a single point in a three dimensional space $^{8}$, where the axes are labeled $l, c$ and $r$. The $l$ coordinate is found by counting all individuals who love Left, and subtracting all individuals who hate Left. The result is then normalized to the size of the population Similarly for the other two coordinates.

Hence,

$$
\begin{aligned}
& l=p_{1}+p_{2}-p_{3}-p_{5} \\
& c=p_{3}+p_{4}-p_{1}-p_{6} \\
& r=p_{5}+p_{6}-p_{2}-p_{4}
\end{aligned}
$$

and $l+c+r=0$.

Note that different distributions of preferences can lead to the same point in the sphere. For instance, the point in the origin is given not only by $p_{1}=p_{2}=\ldots=p_{6}=1 / 6$, but by any combination of preferences such as $p_{1}=p_{3}, p_{2}=p_{5}, p_{4}=p_{6}$.

Note also that this mechanism is very close to defining a Borda count social welfare function.

We can now define the polarization of the preferences in the society as the distance from the center of the sphere:

$$
d(l, r, c) \equiv d\left(p_{1}, p_{2}, \ldots, p_{6}\right)=\sqrt{l^{2}+r^{2}+c^{2}}
$$

Note that $d \in\lfloor 0, \sqrt{2}\rfloor$ : all points thus lie inside a sphere around the origin.

Figure 1 explores how the outcome varies as a function of the distance $d$. The whole range [0,1] is sampled, for all probabilities $p_{1} . . p_{6}{ }^{9}$. When $\pi_{J}-\pi_{H}>0$ a win is assigned to the J-rule; when $\pi_{J}-\pi_{H}<0$ a win is assigned to the H-rule. For each $\operatorname{bin}^{10}$, the frequency of wins with each rule is computed (Figure 1a). The average values of $\pi_{J}$ and $\pi_{H}$ are shown in Figure $1 b$.

\footnotetext{
${ }^{8}$ Florentin Paladi helped in defining this aggregation procedure

${ }^{9}$ The step considered for creating all combinations of probabilities is 0.025 .

${ }^{10}$ The bin width used in the figure is 0.025
} 
Exactly in the center of the sphere the two rules lead to the same payoff, independently of the underlying distribution of preferences. Close to the center, each rule wins in about $50 \%$ of the cases. Then, as we move away from the center the J-rule improves its performance, and is always better when the preferences are totally polarized.

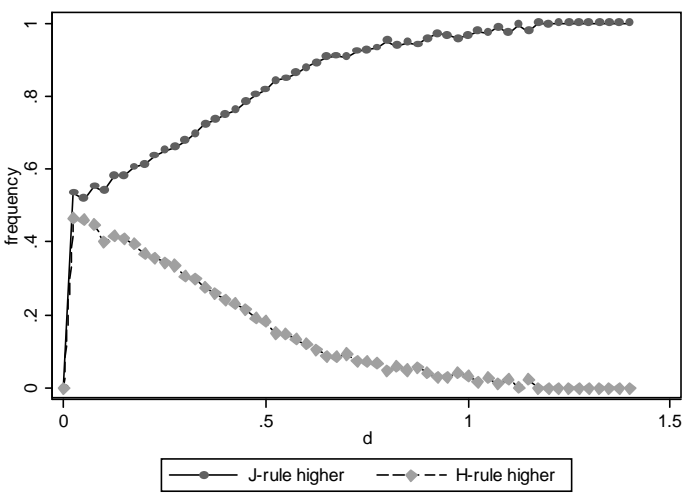

(a)

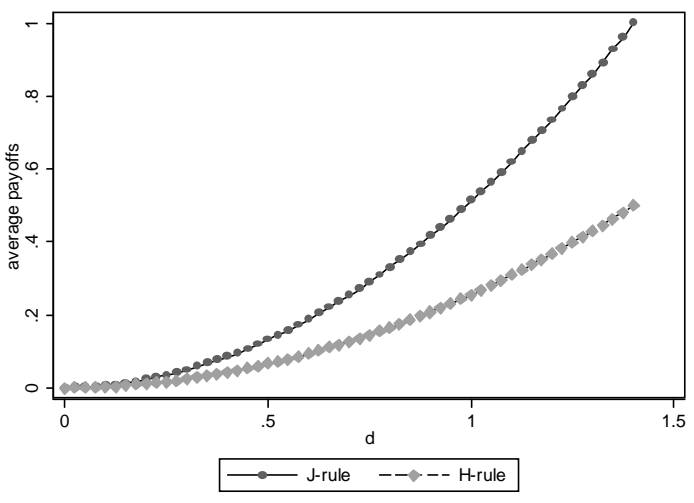

(b)

Figure 1: Frequency of negative and positive values of $\pi_{J}-\pi_{H}$ (a) and average values for $\pi_{J}$ and $\pi_{H}$ (b).

In conclusion, I have shown that, depending on the underlying distribution of preferences, both rules can be optimal. However, as the preferences become more polarized, the J-rule clearly takes the lead.

\subsection{Coefficient of variation}

The variances $\sigma_{J}^{2}$ and $\sigma_{H}^{2}$ are defined for each discrete distribution $D \equiv J, H$ with the expectation (mean) value $\pi_{D}$ as follows:

$$
\sigma_{D}^{2}=\sum_{i=1}^{6} p_{i}\left(\pi_{i, D}-\pi_{D}\right)^{2}
$$

where

$$
\begin{array}{ll}
\pi_{1, J}=p_{1}+p_{2}-p_{3}-p_{4} & \pi_{4, J}=p_{3}+p_{4}-p_{5}-p_{6} \\
\pi_{2, J}=p_{1}+p_{2}-p_{5}-p_{6} & \pi_{5, J}=-p_{1}-p_{2}+p_{5}+p_{6} \\
\pi_{3, J}=-p_{1}-p_{2}+p_{3}+p_{4} & \pi_{6, J}=-p_{3}-p_{4}+p_{5}+p_{6}
\end{array}
$$

and

$$
\begin{array}{ll}
\pi_{1, H}=\left(p_{1}-p_{3}-p_{5}+p_{6}\right) / 2 & \pi_{4, H}=\left(-p_{1}+p_{2}+p_{4}-p_{6}\right) / 2 \\
\pi_{2, H}=\left(p_{2}-p_{3}+p_{4}-p_{5}\right) / 2 & \pi_{5, H}=\left(-p_{2}+p_{3}-p_{4}+p_{5}\right) / 2 \\
\pi_{3, H}=\left(-p_{1}+p_{3}+p_{5}-p_{6}\right) / 2 & \pi_{6, H}=\left(p_{1}-p_{2}-p_{4}+p_{6}\right) / 2
\end{array}
$$


Since variance is scale-sensitive however, it makes little sense to use it as a measure of dispersion when the mean values can significantly differ. I thus divide the standard deviation by the mean to obtain the coefficient of variation, which is scale-free.

Figure 2a shows an interesting result: although in general different, when the two coefficient of variations are conditioned on the distance $d$ they give exactly the same value. On average however, when one rule is better in terms of higher expected payoffs it is also better in terms of lower inequality (panels $b$ and $c$ ).

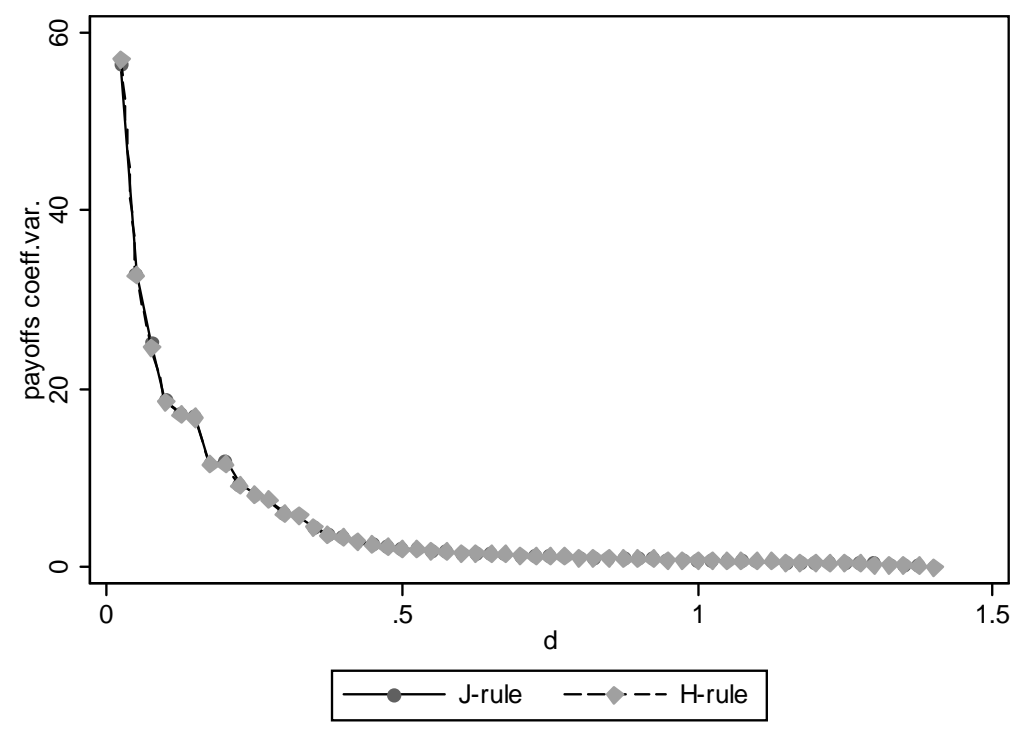

(a) all sample

For any level of fragmentation of preferences, the two rules give the same average value of the coefficient of variation

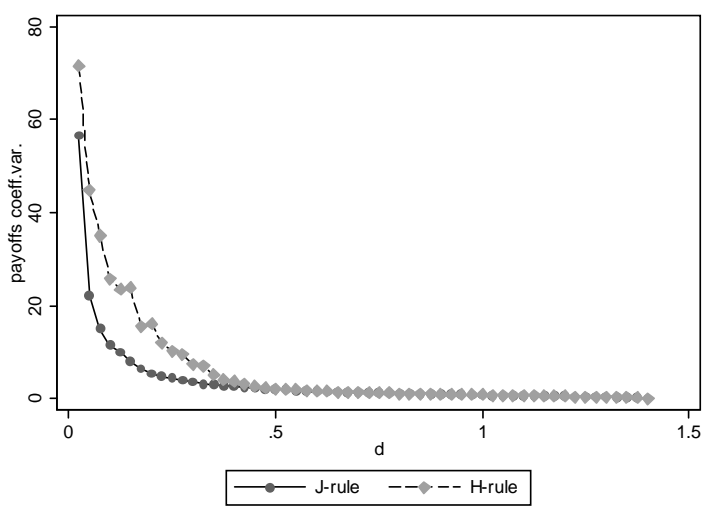

(b) $\pi_{J}>\pi_{H}$

When the J-rule gives higher payoff, it also grants lower inequality

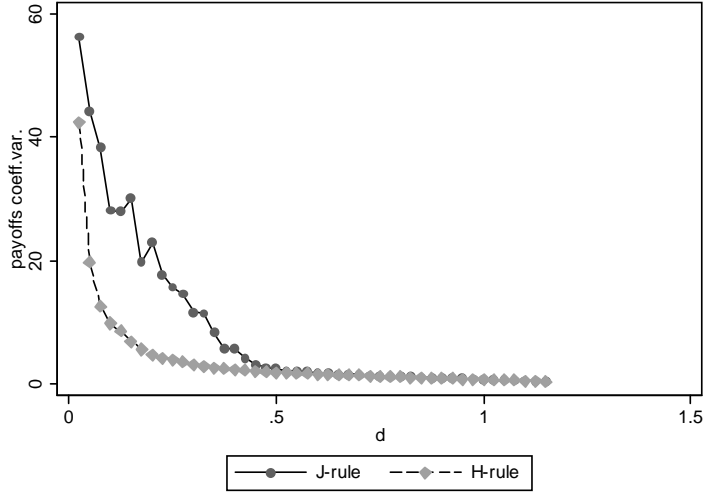

(c) $\pi_{J}<\pi_{H}$

When the J-rule gives lower payoff, it also grants higher inequality

Figure 2: Average value of the coefficient of variations 


\section{From moral to social norms}

Suppose now that all individuals act according to the following strategy: "if nobody acted to you, play according to the H-rule; otherwise do what your last opponent did to you". This rule is reminiscent of the "tit-for-tat" strategy, with the only difference that the reciprocal behavior cannot be targeted to specific individuals. Thus, retaliation is directed towards society in general. For this reason it is labelled Blind tit-for-tat (BT).

It is easy to see that this strategy always leads to the selection of a single action. Which action will actually be selected depends on the distribution of preferences in the population and on the (random) order of interactions. To investigate the selection process, I draw randomly 4 out of 6 probabilities, say $p_{a} . . p_{d}$. We then set $p_{e}=0$ and the remaining probability $p_{f}=1-\left(p_{a}+p_{b}+p_{c}+p_{d}+p_{e}\right)$. I consider, for computational reasons, a slightly modified version of the model, where each person interacts as active player with only one passive player, randomly chosen. I consider 600 individuals and simulate $^{11}$ all interactions for 1,000 periods - an amount of time generally sufficient given the population size - for the selection process to take place. I perform 50 runs with the same parameters, and then consider the average of the frequencies of each action being played at $t=1,000$. I then update the parameters by increasing $p_{e}$ of a 0.01 step, and decreasing $p_{f}$ accordingly. I repeat the process until $p_{f}=0$. Figure 3 shows the results for $p_{2}=0.22 \overline{3} ; p_{3}=p_{6}=0 ; p_{4}=0.1 \overline{3} ; p_{1} \in[0,0.76]$ and $p_{5}=1-\sum_{i \neq 5} p_{i}$. As $p_{5}-$ which corresponds to people loving Right and hating Left - increases, the probability that Left is selected decreases and the probability that Right is selected increases. No threshold effects are present. Such smooth transitions are observed also for other combinations of the parameters. ${ }^{12}$

Note that a $50 \%$ probability that one action is selected does not mean that half of the population plays that action, while the other half plays something else. It means that in $50 \%$ of the runs, without changing the parameters, that action is played by all individuals, while in the other $50 \%$ of the runs some other action is selected as the only action being played.

\footnotetext{
11 We develop an agent-based simulation using the open-source JAS platform (http://jaslibrary.sourceforge.net), see Sonnessa (2004).

${ }^{12}$ In the companion paper (Richiardi, 2005) I show analytically that in the range $(0,1)$ the fraction $\alpha$ of the population playing any action is a random walk. The steps of the random walk are a function of $\alpha$.
} 


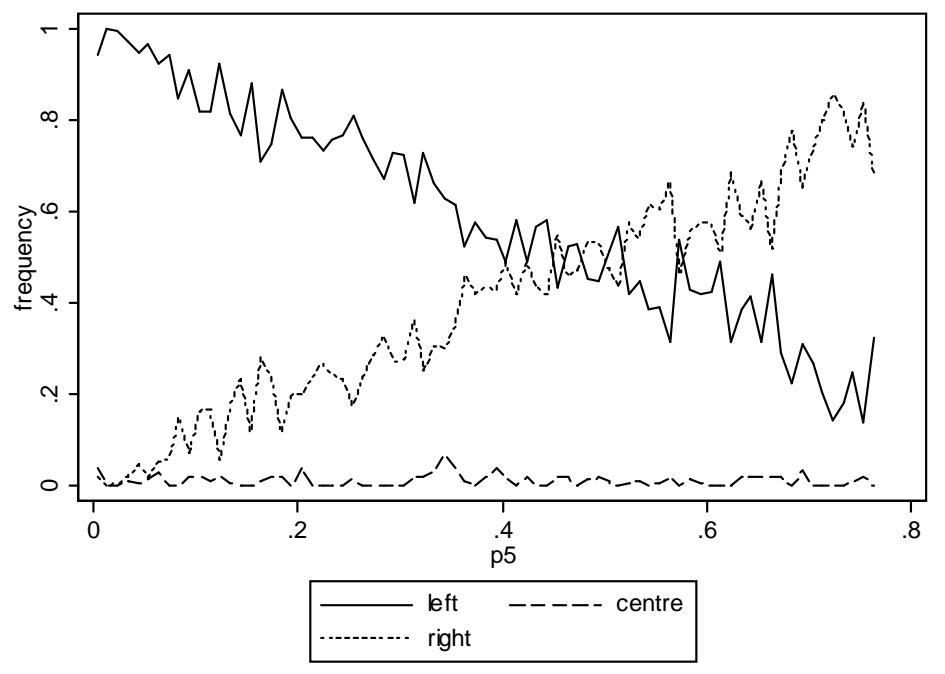

Figure 3: Frequency of each action being played after 1,000 periods, BT-rule, four probabilities fixed, average of 50 runs for each set of the parameters.

Note that this process of path dependency closely resembles the creation of a social norm, which prescribes to play one single action, irrespective of individual preferences. Should we have two distinct populations with the same distribution of preferences, it is very likely that we could observe the selection of a different action within each population, as the social norm of that community. In fact, it is well known that the existence of social norms creates conformity within groups and heterogeneity across groups (Gintis, 2003).

An interesting question is whether the selection of a single action leads to higher or lower average payoff, and to higher or lower variance. Figure 4 plots the evolution of the average payoff, from a situation where everyone plays according to the H-rule (up to $t=$ 100 ), to a situation where everyone plays according to the BT-rule (from $t=100$ onward). After a period of oscillations the system eventually settles down and a single action is played by all individuals in the population. In the particular case depicted in Figure 4, the average payoff actually increases, in the stationary state (although during some parts of the transition process it is actually lower). The upper and lower bounds in the figure are computed as

$$
\begin{aligned}
& \text { average payoff lower bound }=m_{\pi}-1.96 s_{\pi} \\
& \text { average payoff upper bound }=m_{\pi}+1.96 s_{\pi}
\end{aligned}
$$

where $m_{\pi}$ and $s_{\pi}$ are respectively the mean and the standard deviation of the average payoff under the moral norm regime, i.e. in the first 100 periods. $^{13}$

\footnotetext{
${ }^{13}$ By the law of large numbers any statistics on the population, for a given distribution of preferences, is a Gaussian random variable. Thus, approximately $95 \%$ of the observations should lie in the interval between the upper and the lower bound. If we observe a realization outside the interval after having
} 


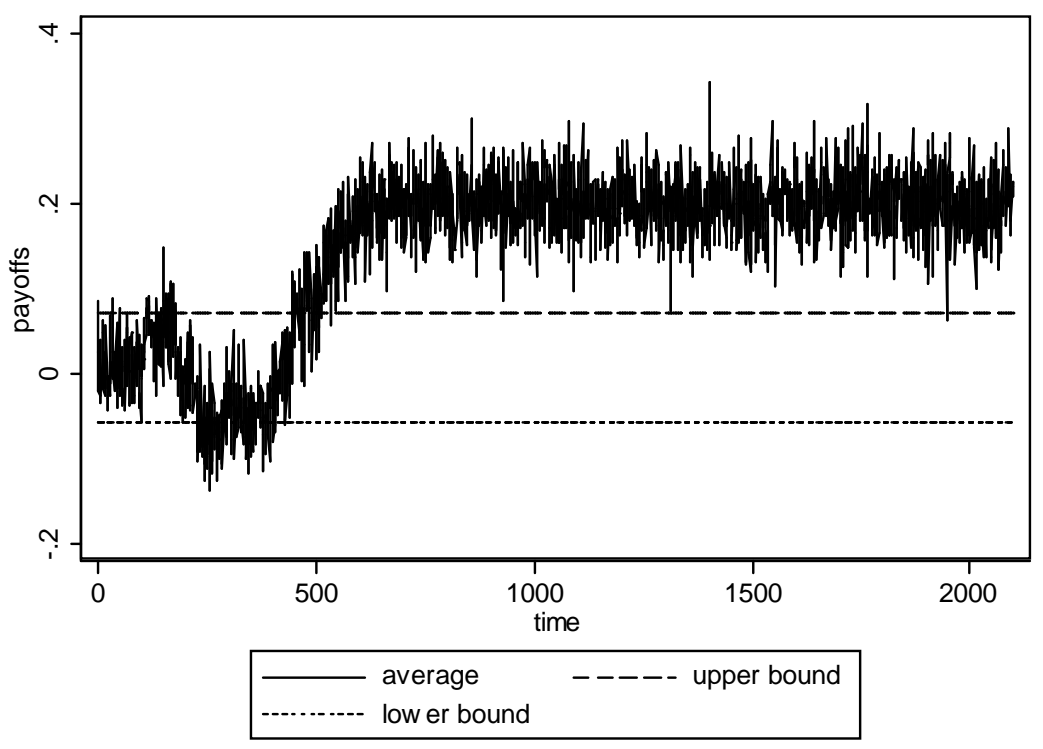

Figure 4: From moral to social norms. Up to $t=100$ everybody plays according to the H-rule. From $t=$ 100 onward everybody plays according to the BT-rule. After a period of oscillations, one action is selected. Upper and lower bounds are computed using the average of the mean and the standard deviation of the payoffs during the H-rule regime.

Table 2 reports the frequency when the mean and variance under the tit-for-tat strategy are higher (lower) than under the J-rule and H-rule, respectively. 100 runs are performed starting with the J-rule, and 100 runs are performed starting with the H-rule. Preferences are distributed randomly. During the first 100 periods of each run, every individual in the population follows the moral norm (either the J-rule or the H-rule). From $t=100$ onward, everybody plays according to the BT-rule. The first 1,000 periods under the BT regime are discarded ${ }^{14}$. Then, the average of the mean and variance of the payoffs in the last 1,000 periods are compared with the corresponding significance interval computed when everybody played according to the moral norm. This interval is computed according to equation (6) for the mean, and to equation (7) for the variance:

$$
\begin{aligned}
& \text { variance lower bound }=m_{\sigma^{2}}-1.96 s_{\sigma^{2}} \\
& \text { variance upper bound }=m_{\sigma^{2}}+1.96 s_{\sigma^{2}}
\end{aligned}
$$

where $m_{\sigma^{2}}$ and $s_{\sigma^{2}}$ are respectively the mean and the standard deviation of the payoff variance under the moral norm regime, i.e. in the first 100 periods.

changed the rules of behaviour, we can then conclude that we are sampling from a different distribution: the statistics (here, the average payoff or the variance) has significantly changed.

${ }^{14}$ This is generally sufficient for the selection of one single action, which is played by everyone in the population. 


\begin{tabular}{|c|c|c|c|c|c|}
\hline & \multicolumn{2}{|c|}{ (social norm established*) } & & \multicolumn{2}{|c|}{ (social norm established*) } \\
\hline & $\begin{array}{l}\% \text { higher than } \\
\text { moral norm }\end{array}$ & $\begin{array}{l}\text { \% lower than } \\
\text { moral norm }\end{array}$ & & $\begin{array}{l}\% \text { higher than } \\
\text { moral norm }^{(+)}\end{array}$ & $\begin{array}{l}\text { \% lower than } \\
\text { moral norm }\end{array}$ \\
\hline$\pi_{J}$ & .58 & .42 & $\sigma_{J}^{2}$ & .98 & .02 \\
\hline$\pi_{H}$ & .34 & .39 & $\sigma_{H}^{2}$ & .98 & .00 \\
\hline
\end{tabular}

Table 2: Moral and social norms compared

Table 2 shows that almost can happen with respect to the average payoff. The selection of a single strategy under the tit-for-tat regime leads to a significant increase in the average payoff in $58 \%$ of the runs, and to a significant decrease in $42 \%$ of the runs, when compared with the J-rule. When compared with the H-rule, it leads to a significant increase in the average payoff in $34 \%$ of the cases, to a significant decrease in $39 \%$ of the cases, and to results that are roughly similar in $27 \%$ of the cases. However, playing BT leads almost always to an increase in the variance of the payoffs, hence to an increase in the degree of inequality in the population. This is rather intuitive: when only a single action (the social norm) is played in a population with heterogeneous preferences, someone will be very happy, while someone else very unhappy.

\section{From social norms to moral outcomes}

So far, I have compared situations where everybody played according to the same strategy, i.e. either following a moral norm (the J-rule or the H-rule) or following the titfor-tat rule. Now, it is interesting to see what happens when the "blind tit-for-tat" guys are mixed together with the "moral" individuals. Are we going to observe a proportionally "mixed" outcome? And if not, are a few BT reciprocators enough to disrupt the moral order, or, conversely, a few fellows of Jesus and Hillel are sufficient to "redeem" the entire population? The observation that reciprocity is indeed one of the pillars of human societies suggests that the most relevant case is when a (possibly small) bunch of "moral" individuals are introduced in a BT population.

I look at the fraction of the entire population that has to play according to the moral norm in order to have an outcome (in terms of average payoff and variance) not significantly different to the one obtained when everybody plays according to the moral norm. Table 3 reports, for different values of this fraction, the frequency when the outcome for the statistics considered is within the significance interval, as defined above (mean \pm 1.96 std. dev., computed when everybody plays the moral norm). About 2,000 simulation runs are performed. Preferences are randomly distributed, but are held constant while varying the fraction of the population that plays according to the moral norm. 


\begin{tabular}{cccccc}
\hline $\begin{array}{c}\text { Fraction of the } \\
\text { population playing } \\
\text { the moral norm }\end{array}$ & Runs & \multicolumn{2}{c}{ J-rule } & \multicolumn{2}{c}{ H-rule } \\
\hline & & $H_{0}: \pi=\pi_{J}$ & $H_{0}: \sigma^{2}=\sigma_{J}^{2}$ & $H_{0}: \pi=\pi_{H}$ & $H_{0}: \sigma^{2}=\sigma_{H}^{2}$ \\
\cline { 3 - 6 } $100.0 \%$ & 560 & $97.7 \%$ & $100.0 \%$ & $100.0 \%$ & $100.0 \%$ \\
$5.0 \%$ & 560 & $96.8 \%$ & $99.8 \%$ & $98.9 \%$ & $99.6 \%$ \\
$1.0 \%$ & 560 & $78.3 \%$ & $83.2 \%$ & $77.0 \%$ & $84.1 \%$ \\
$0.5 \%$ & 314 & $56.3 \%$ & $65.9 \%$ & $49.4 \%$ & $63.1 \%$ \\
\hline
\end{tabular}

Table 3: Non-refusal of the hypothesis that the mean and variance in the payoffs are equal to the case when everybody plays according to the moral norm $\left(H_{0}\right)$, different fractions of the population departing from the moral norm considered.

When only $5 \%$ of the population plays according to the moral norm, the outcome is not significantly different from that occurred when everybody shared the moral norm in $96.8 \%$ of the cases for the J-rule, and in $98.9 \%$ of the cases for the H-rule. A small fraction of $1 \%$ of "moral" guys is sufficient to guarantee the same result as in the "moral" society three quarters of the times!

\section{Conclusions}

Preferences lie at the foundations of economics. The literature on reciprocity and the emergence of social norms generally makes the assumptions that preferences, or at least some proxy, are observable. Individuals can thus decide whether to be keen toward their neighbours or not. Conversely, the case when preferences are not observable has received little or no attention at all in the scientific literature. This is surprising, especially because the theme is at the hearth of the western religious literature. This paper provides a very simple model of individual interaction, in order to test the implications in terms of aggregate welfare of two well-known moral norms: the socalled golden rules of Jesus ("do to your neighbours what you would like them do to you") and the prescription by Hillel ("don't do to your neighbours what you would not like them do to you"). I consider them as an idealization of an imperative and a more liberal approach to social norms. I find that the aggregate welfare depends on the distribution of preferences in the society. When the preferences are highly fragmented, the two rules give roughly the same expected payoff; However, as the preferences become more polarized, the fraction of combinations favourable to the J-rule increase, and reaches $100 \%$ when the preferences are totally polarized. So, the J-rule turns out to stochastically dominate the H-rule, for an unknown distribution of preferences. ${ }^{15}$

A third, more realistic behavioural rule is then introduced, a "blind tit-for-tat" strategy that prescribes "do to your neighbours what they have done to you". I show that if this strategy is followed by everybody it leads to the selection of a single behaviour, which becomes established as a social norm. This behaviour leads in general to more inequality, with respect to the Jesus or Hillel rules. However, it is sufficient that a small

\footnotetext{
${ }^{15}$ In Richiardi (2005) I suggest another opearationalization of the H-rule, which turns out to be always better than the J-rule proposed here.
} 
group (about 1\%) of the population keeps on playing one of the two moral norms to recover the same social welfare that is obtained when everybody plays the moral norm.

\section{References}

Axelrod R., Hamilton W.D. (1981), "The evolution of cooperation", Science, 211, 1390-1396

Boyd R., Richerson, P.J. (1985), Culture and the Evolutionary Process, University of Chicago Press, Chicago

Cavalli-Sforza L., Feldman M.W. (1981), Cultural Transmission and Evolution, Princeton University Press, Princeton, NJ

Fehr E., Fischbacher U. (2004), "Social Norms and Human Cooperation", TRENDS in Cognitive Sciences, 8 (4), 185-190

Gintis H. (2000), "Strong Reciprocity and Human Sociality", Journal of Theoretical Biology, 206, 169-179

Gintis H., Bowles S., Boyd R., Fehr E. (2003), "Explaining Altruistic Behavior in Humans", Evolution and Human Behavior, 24, 153-172

Lumsden C.J., Wilson E. O. (1981), Genes, Mind, and Culture: The Coevolutionary Process, Harvard University Press, Cambridge, MA

Richiardi M. (2005), Jesus vs. Hillel. Moral and Social Norms in Heterogeneous Populaitons, LABORatorio R. Revelli Working Paper No. 40.

Simon H.A. (1993), “Altruism and economics", American Economic Review, 83, 156161.

Sober E., Wilson D.S. (1998), Onto Others: The Evolution and Psychology of Unselfish Behavior, Harvard University Press, Cambridge, MA

Sonnessa M. (2004), "JAS: Java Agent-based Simulation Library, an Open Framework for Algorithm-Intensive Simulations", in Contini B., Leombruni R., Richiardi M. (eds), Industry and Labor Dynamics: The Agent-Based Computational Economics Approach, World Scientific, Singapore

Trivers R. L. (1971), “The evolution of reciprocal altruism”, Quarterly Review of Biology, 46, 35-57.

Wilson D.S., Dugatkin L.A. (1997), "Group selection and assortative interactions", American Naturalist, 149, 336-351. 